\title{
POLICY The drivers and impact of emergency care reconfiguration in Ireland: Results from a large mixed-methods research programme
}

\author{
Author: John P Browne ${ }^{\mathrm{A}}$
}

\begin{abstract}
Ireland, like many countries, has reconfigured emergency care in recent years towards a more centralised model. Although centralisation is presented as 'evidence-based', the relevance of this evidence is challenged by groups which hold values beyond those implicit in the literature. The Study of the Impact of Reconfiguration on Emergency and Urgent Care Networks (SIREN) programme was funded to evaluate the development and performance of emergency and urgent care systems in Ireland. SIREN found that the drivers of reconfiguration in Ireland are based on safety and efficiency claims which are highly contestable. Reconfiguration was not associated with improvements in safety or efficiency and may have exacerbated the growing capacity challenges for acute hospitals. These findings are consistent with UK research. Our study adds to an emerging literature on the interaction between a narrow technocratic approach to health system planning and the perspectives of the public and patients.
\end{abstract}

KEYWORDS: Emergency care, smaller hospitals, Ireland

\section{Context}

Ireland is a country of $70,200 \mathrm{~km}^{2}$ on the west of Europe with a population of 4.8 million in $2016 .{ }^{1}$ This is projected to rise to as much as 6 million people by $2030 .^{2}$ While there is uncertainty over the exact geographical distribution of this growth, all regions, including those outside the main urban centres, will see an absolute rise. ${ }^{3}$ It is also projected that older age groups will see a disproportionate rise. ${ }^{2}$ This will substantially increase demand for public hospital services, for example, emergency department (ED) attendances are projected to increase by as much as $26 \%$ by $2030 .^{2}$

These demographic changes are occurring at a time of stress on the public hospital service. ED overcrowding, as measured by patients waiting for hospital admission on trolleys, is now a yearround phenomenon, particularly at the major regional hospitals. Some of this stress is due to demographic pressure and some is related to resource constraints. ED attendances and emergency

Author: ${ }^{\text {A }}$ rofessor of health services research, University College Cork, Cork, Ireland admissions have increased in the last decade, while staff and acute bed numbers have fallen. ${ }^{5-7}$ Ireland now has a very low stock of hospital beds by international standards and a bed occupancy rate of $95 \%$, well above the Organisation for Economic Co-operation and Development average of $76 \% .{ }^{8}$ The number of inpatient public hospital beds will have to increase by 25 to $48 \%$ by 2030 simply to maintain the already low per capita bed ratio. ${ }^{8}$ Public healthcare in Ireland is managed and delivered by the Health Service Executive (HSE). Eligibility is complex and operates through multiple income, age and health criteria. Patients who do not meet these criteria must pay to get a referral from their general practitioner (GP) to attend a public ED. If they go straight to the ED without a referral they must pay a fixed charge of $€ 100$. Thus, even before geographic variation is taken into account, there are considerable financial barriers to accessing public healthcare.

\section{Policy history}

Like many other developed countries, Ireland has experimented with the reconfiguration of acute hospital services, particularly the centralisation of specialist services at fewer hospitals, serving a higher volume of patients. Emergency care reconfiguration was a pillar of a 2006 system-wide policy to 'transform' healthcare delivery in the then newly established HSE. ${ }^{9}$ Prior to reconfiguration, 35 hospitals operated full 24/7 EDs for all age groups, and a further three children's hospitals had 24/7 EDs. A part of the reconfiguration programme involved closing or 'redesignating' EDs at smaller hospitals to urgent care centres and local/minor injury units. Ten EDs were closed or re-designated, seven of which were in towns outside the main urban centres. ${ }^{10}$ The reconfiguration programme received impetus from various patient safety investigations, and these informed a 2013 policy document on the role of smaller hospitals which reinforced the rationale for ongoing reconfiguration. ${ }^{11}$ Details of the ED reconfiguration programme are shown in Fig 1.

Ireland now has different models of ED provision in different regions, and these variations do not seem to be related to population or underlying geographical constraints. The midwestern region, for example, has a single public ED for a population of 385,172 living in a largely rural geographic area of $8,252 \mathrm{~km}^{2}$. County Dublin, by contrast, has nine public EDs for a population of 1,345,402 (1 per 149,489 people) living in a largely urban area of $922 \mathrm{~km}^{2}$. There are indications from senior clinicians and 
Fig 1. Timeline of emergency department changes. $E D$ = emergency department; LIU = local injuries unit; $\mathrm{UCU}=$ urgent care unit.

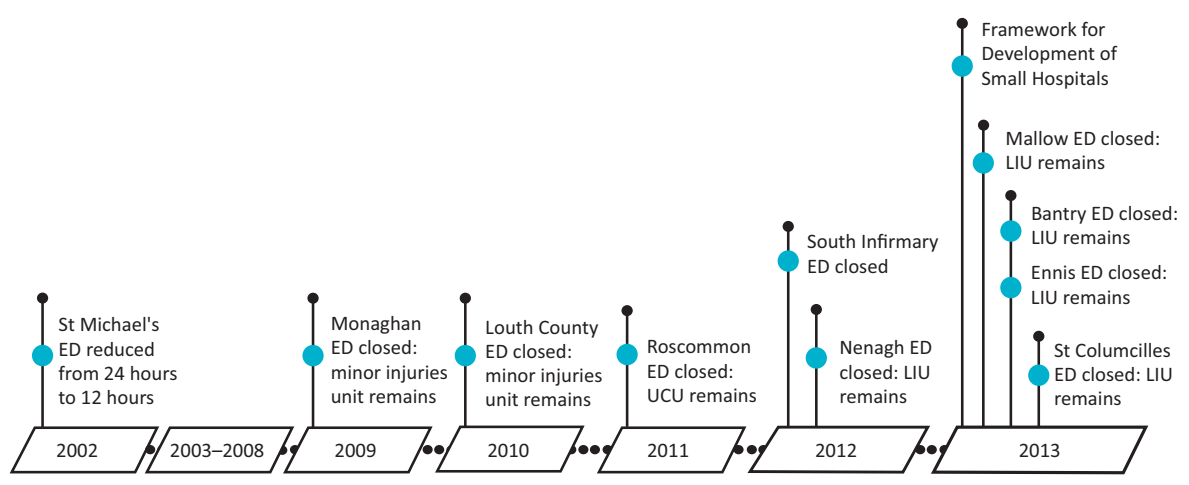

administrators in the HSE that the ED reconfiguration programme has not ended and as many as nine other EDs are under explicit threat of downgrade. Public opposition since 2013 has managed to resist further downgrades but there are fears of a 'stealth downgrade' programme in the remaining smaller hospitals with 24/7 EDs. These EDs often have little consultant cover out-of-hours, onerous rostering arrangements and a deteriorating infrastructure.

\section{The SIREN research programme}

In 2013 the Study of the Impact of Reconfiguration on Emergency and Urgent Care Networks (SIREN) research programme was funded to evaluate the development and performance of emergency and urgent care systems in Ireland. This paper summarises its main findings, with a focus on the role of EDs in smaller hospitals. The paper also presents the personal views of the principal investigator about policy implications.

SIREN used a mixed-methods design and has produced nine peer reviewed publications to date. These publications were based on a consensus development exercise with 17 national experts to identify appropriate indicators of system performance; a qualitative interview study with 175 purposively selected internal and external stakeholders across all regions; a qualitative analysis of the major Irish policy documents in this field; a national analysis of case-fatality ratios for serious emergency conditions before and after reconfiguration over the period 2002 to 2014; an analysis of changes in acute hospital capacity and ED overcrowding over the period 2005 to 2015 in four regions which had undertaken reconfiguration; an ecological analysis of the drivers of potentially avoidable emergency admission across all 26 counties; a crosssectional analysis of geographic variation in resources for urgent and emergency care in the year 2014; and a cross-sectional survey of the experience of 1,205 patients who had used urgent and emergency care services in the year 2015. ${ }^{9,10,12-17}$ The term 'internal stakeholders' refers to HSE clinicians and managers who were involved, to varying degrees, in the reconfiguration process. 'External stakeholders' were a heterogeneous group of GPs, pre-hospital care providers, civic groups opposed to reconfiguration, private hospital representatives, local politicians and media members.

\section{What drove reconfiguration in Ireland?}

The drivers of reconfiguration were similar to those seen in the UK. ${ }^{18}$ Internal stakeholders were convinced of the poor efficiency and safety of emergency care at lower volume hospitals, and disparaging of the role of community groups and local politicians in campaigning to resist reconfiguration. External stakeholders focused on the harms associated with poor access to care and the loss of a community resource. The general impression was of a confused public discourse; internal stakeholders put forward an incoherent and inconsistent line of argument which external stakeholders reacted to with suspicion. The terms of engagement between both groups were unclear, and as a result there seemed to be little opportunity for meaningful dialogue. This problem can be illustrated by analysing the four main drivers of reconfiguration separately.

\section{Safety}

The most commonly cited driver of reconfiguration was concern about the safety of emergency care at smaller hospitals. Internal stakeholders stated that this concern was based on 'international evidence' about the relationship between volume and outcome. ${ }^{9}$ However, this evidence only covers a small number of conditions, such as major trauma and stroke, most of whom already bypass or are transferred from smaller hospitals. The evidence, therefore, maps poorly to the policy arena because it does not speak to the majority of patients that are impacted when EDs are closed or downgraded. ${ }^{10}$ There was also a sense that smaller EDs were not 'proper' by international standards as they had insufficient on-site support from surgery, anaesthesia and intensive care, and variable out-of-hours consultant cover. Some internal clinical stakeholders were less inclined to agree with broad generalisations about the safety of smaller EDs. They argued that regulatory investigations had been used to drive reconfiguration across the country without evaluating each hospital on a case-by-case basis. There was concern that ED closures could lead to unanticipated safety issues because of the higher volume of patients attending hub hospitals after reconfiguration and the disruption caused to historical relationships between smaller hospitals and primary care.

\section{Efficiency}

Internal stakeholders believed that there were too many EDs in Ireland and the resources required were unsustainable within current budget constraints. A SIREN analysis of resource allocation for emergency care in Ireland found some support for the contention that EDs tend to become less efficient when they serve smaller populations. ${ }^{16}$ However, this study also found that reconfigured regions have higher pre-hospital expenditure to compensate for fewer hospital-based services. External 
stakeholders were sceptical about the likelihood of efficiency gains, listing a range of unanticipated costs and administrative wastage that they had observed post reconfiguration. ${ }^{9}$ It is noteworthy that most ED reconfiguration in Ireland has occurred in rural areas, where significant investment in pre-hospital services is required to alleviate the harms associated with reduced access. Reconfiguring services in urban areas, particularly Dublin where nine EDs continue to operate in close proximity to each other, would appear to offer greater scope for efficiency gains, without necessarily affecting the timeliness of access. Yet internal stakeholders made little reference to the duplication of emergency services in Dublin.

\section{Access}

From the perspective of internal stakeholders, the stated goal of reconfiguration was to design a hub-and-spoke model that delivered patients as quickly as possible to a facility that was 'appropriate' for the severity of their condition. The centralisation of cancer services was invoked as a success story to which emergency care should aspire, but cancer care does not have the same time-sensitive geographical constraints as emergency care. External stakeholders highlighted the problems with imposing a hub-and-spoke model on different geographies, and the fact that smaller hospitals were capable of providing high-quality care for most patients requiring time-sensitive care, particularly older patients with conditions that are best managed closer to their homes. ${ }^{9}$ The role of geography in designing Ireland's regional emergency care systems was not clear. Factors such as rurality played a role in some regions, but these factors were applied inconsistently across the country. ${ }^{10}$ For example, the major Dublin hospitals continue to provide much of the critical care for counties in the north-east, midlands, and south-east regions despite being at the extreme periphery of those regions.

\section{Community values and local politics}

External stakeholders stressed the tangible (eg economic growth) and intangible (eg community self-esteem) benefit of hospital services to a locality. This was not accepted by some internal stakeholders who stated that the determining factor in the viability of an ED should be whether it was 'fit for purpose'. There was a notable degree of cynicism from both internal and external stakeholders regarding the motivations of local politicians when resisting ED closures. It was evident from an analysis of the pre-reconfiguration consultation process that genuine, ongoing and inclusive engagement by planners with communities was rare. Many stakeholders also acknowledged the role of 'medical politics' in the policy process, for example, it was stated that some reconfiguration plans were biased towards the training and research demands of medical schools rather than local population needs and had disrupted historical relationships between hospitals. It was also evident from an analysis of policy documents that senior clinicians at the larger teaching hospitals had a strong influence on the reconfiguration agenda. ${ }^{9}$

\section{What was the impact of emergency care reconfiguration in Ireland?}

The introduction of the reconfiguration programme coincided approximately with a small reduction in the rate of improvement in case fatality for serious emergency conditions. The national annual percentage change in case fatality for a basket of 16 conditions over the period 2002 to 2014 was a decrease of $2.1 \%$.
The decline was faster from 2002 to 2007 (annual percentage change $-3.4 ; 95 \%$ confidence interval (CI) -4.4 to -2.4 ), compared to 2007 to 2014 (annual percentage change -1.2; $95 \%$ CI -1.9 to -0.5$)$. No distinct pattern of change was identified among regions which underwent substantial reconfiguration of emergency services compared to those that did not. ${ }^{14}$ Six counties had a statistically significant improvement in case fatality over the period 2007 to 2014 . One of these was Dublin, and the other five (Donegal, Kerry, Louth, Mayo and Sligo) were counties which retained a smaller ED throughout the reconfiguration process. However, this was a limited study which was not capable of making strong inferences about the impact of reconfiguration on clinical outcomes. The most that one can infer confidently is that there is no obvious signal of harm or benefit in the case fatality data. Plausible explanations for the slowing rate of improvement from 2007 to 2014 include a floor effect, and the impact of Ireland's 2008 economic recession which resulted in decreases in funding and staff across the public healthcare service. The patient experience survey was a snapshot of the situation in early 2015 and does not provide a 'before and after' analysis of the impact of reconfiguration. The survey found no association between the type of emergency care model adopted in different regions and self-reported care experience. ${ }^{17}$ Overall, $36 \%$ of service users in the SIREN survey rated their care as excellent, slightly lower than figures from comparable studies conducted in the UK where ratings of $39 \%$ and $41 \%$ were reported. The only significant finding was that service users in the mid-west region, which had shut three EDs and centralised care to one tertiary hospital, reported significantly lower overall ratings of care than those in the Dublin north-east region, which has undertaken little change.

The SIREN analysis of demand and capacity changes in regions which had undergone reconfiguration, found a substantial rise in emergency admissions over the period 2005 to 2015 without a commensurate increase in hospital beds. ${ }^{4}$ In three of the studied regions, the immediate impact of reconfiguration on ED overcrowding was either non-significant or associated with a short-term 'shock' to observed trolley numbers followed by convergence with the pre-reconfiguration trend over the following 12 months. In one of the regions, a longer-term increase in the overcrowding trend was observed. These results suggest that it is possible for regional care systems to absorb the loss of a smaller ED in the short-term. However, more recent data on overcrowding suggests this is likely to have long-term negative impacts in the hospitals which are expected to absorb the impact of ED closures. For example, the tertiary hospitals which serve the southern and mid-west regions, both of which have had to absorb the impact of ED closures in neighbouring hospitals, have seen a doubling of the number of patients waiting on trolleys for a hospital bed over the period 2016 to $2018 .{ }^{19}$ There is also little evidence that initiatives that focus on "potentially avoidable' emergency admissions (by, for example, improving the primary care management of certain chronic conditions and providing alternative care pathways when these conditions are exacerbated) can significantly reduce the demands on acute hospitals. A SIREN analysis of the impact of population and health system factors on county-level variation in potentially avoidable emergency admissions in Ireland found that admission rates are driven by area level deprivation, hospital admission policy and private health insurance coverage, and do not seem to have a strong association with primary care resources. ${ }^{15}$ 


\section{Summary of SIREN findings}

The drivers of reconfiguration in Ireland are the same as in the UK, and there is the same confusing and dysfunctional dialogue between planners and the public. ${ }^{18}$ Reconfiguration was not associated with improvements in safety or efficiency and may have exacerbated the growing capacity challenges for acute hospitals. These findings are also consistent with UK research. A 2019 UK study, which is the only other to examine the impact of ED closure on a broad set of emergency conditions, found a small but statistically significant increase in case fatality following ED closure, with the caveat that the increase may have been caused by changes in admissions. ${ }^{20}$ A 2015 review of 123 proposed reconfiguration projects in the UK found no evidence of significant savings resulting from reconfiguration. ${ }^{21}$ Finally, a 2014 UK study found that most of the variation in potentially avoidable emergency admissions is due to factors such as socioeconomic deprivation, which are beyond the control of health services. ${ }^{22}$

\section{Policy implications}

These findings point to the need for a pause to the ongoing centralisation of public emergency care services in Ireland. Policy makers should recognise the potential harms of centralisation and end their narrow focus on safety concerns in the highest risk patients. Ireland is in the midst of an escalating capacity crisis in acute hospitals caused by previous cutbacks and a growing and ageing population. Smaller hospitals can provide care close to home for the growing number of older patients with recurrent care needs arising from long-term conditions. Smaller hospitals can also provide safe emergency care to most patients if structured properly. For example, a 2019 Irish study on the volume-outcome relationship in emergency abdominal surgery found identical results in low- and high-volume centres. ${ }^{23}$ This was in part because many low-volume centres were served by high-volume surgical teams. Unfortunately, the study was presented in the Irish media as supporting the case for reducing the number of hospitals providing critical surgery services. ${ }^{24}$

Evidence production, selection and interpretation in this field is suggestive of a bias towards centralisation. Previous justifications for the reconfiguration agenda have suffered from biases in evidence framing (eg by focusing on high-risk patient groups experiencing single episodes of care), selection (eg by focusing on evidence that addresses a subset of policy concerns) and interpretation (eg by presenting confused understanding of risk in different settings). These intellectual vices have been observed in many policy fields and are an important obstacle to developing meaningful dialogue between healthcare providers and the populations they serve. ${ }^{25}$

Dialogue would also improve if advocates of the centralisation policy accepted that involvement by elected representatives in the planning of local healthcare services is not necessarily 'interference'. In a field where there is no clear policy direction from the evidence and unsatisfactory consultation in the past, it should not be surprising that local politicians find it necessary to voice the concerns of the people they represent. The alliances of clinicians and managers promoting centralisation should instead reflect on the role of medical politics in driving the agenda. This includes the tendency of larger teaching hospitals to advocate for policies that will increase their share of resources, and the tendency of professional organisations to promote the preferences of their members such as the desire to work within large academic medical systems. These agendas are not inherently illegitimate but they are not always consistent with population needs.

Evidence is important in this field, but we should also recognise the role played by values. Both sides in the centralisation debate value excellence, but have different understandings of what this means. Proponents focus on professionally driven definitions of what 'proper' emergency care looks like. Opponents focus more on community driven definitions of what patient-centred care looks like. A starting point for developing a functional dialogue would be to compromise and develop a pragmatic understanding of excellence that bridges both sides and can meet the varying needs of populations in different regions.

\section{Conflicts of interest}

Funding for the SIREN research programme was received from the Health Research Board (HRB), Funding University College Cork Collaborative Applied Research Grant 2012 (CARG/2012/28). The HRB had no involvement in data collection, analysis or interpretation; or any aspect pertinent to the study.

\section{Acknowledgements}

SIREN was a collaboration of University College Cork, Trinity College Dublin, the Royal College of Surgeons in Ireland and the Health Service Executive. The following researchers worked on the various SIREN studies: Dr Anthony Fitzgerald, Dr Brenda Lynch, Dr Elsa Droog, Dr Maria Boyce, Dr Conor Foley, Dr Sheena McHugh, Dr Paul Corcoran, Dr Rebecca Dennehy, Siobhan Boyle, Claire Collins and Dr Claire Buckley (University College Cork); Prof Steve Thomas, Dr Bridget Johnston and Dr Bridget Kane (Trinity College Dublin); Prof Susan Smith (Royal College of Surgeons in Ireland); and Dr Orla Healy (Health Service Executive). Dr Leah McClimans of the University of South Carolina provided important insights on the relationship between evidence and values while the manuscript was in development. I also would to thank the members of the project advisory groups for their advice and support.

\section{References}

1 Central Statistics Office. Census 2016 Summary Results - Part 1. Dublin: Central Statistics Office, 2016.

2 Wren MA, Keegan C, Walsh B et al. Projections of demand for healthcare in Ireland, 2015-2030, first report from the HIPPOCRATES Model, Research Series No 67. Dublin: The Economic and Social Research Institute, 2017.

3 Central Statistics Office. Regional population projections 20172036. Dublin: Central Statistics Office, 2019.

4 Lynch B, Browne JP, Corcoran P et al. An interrupted time-series analysis of the impact of emergency department reconfiguration on regional emergency department trolley numbers in Ireland from 2005-2015. BMJ Open 2019;9:e029261.

5 Health Service Executive, Planning and Business Information Unit. Monthly performance reports. Dublin: Health Service Executive, 2008-2017.

6 Health Service Executive, Health Intelligence Unit. Hospital inpatient enquiry health atlas Ireland. Dublin: Health Service Executive, 2017.

7 Thomas S, Burke S, Barry S. The Irish health-care system and austerity: sharing the pain. Lancet 2014;383:1545-6.

8 Keegan C, Brick A, Walsh B et al. How many beds? Capacity implications of hospital care demand projections in the Irish hospital system, 2015-2030. Int J Health Plann Manage 2019;34:e569-82.

9 Droog E, Foley C, Healy $\mathrm{O}$ et al. Perspectives on the underlying drivers of urgent and emergency care reconfiguration in Ireland. Int J Health Plann Manage 2018;33:364-79. 
10 McHugh SM, Foley C, Boyce M et al. Understanding the impetus for major systems change: A multiple case study of decisions and non-decisions to reconfigure emergency and urgent care services. Health Policy 2019;123:728-36.

11 Department of Health and Children, Ireland. Securing the future of smaller hospitals: a framework for development. Dublin: Department of Health and Children, 2014

12 Boyle S, Dennehy R, Healy O, Browne JP. Development of performance indicators for systems of urgent and emergency care in the Republic of Ireland: update of a systematic review and consensus development exercise. HRB Open Research 2019:1;6.

13 Foley C, Droog E, Healy O et al. Understanding perspectives on major system change: A comparative case study of public engagement and the implementation of urgent and emergency care system reconfiguration. Health Policy 2017;121:800-8.

14 Lynch B, Fitzgerald AP, Healy $O$ et al. Case fatality ratios for serious emergency conditions in the Republic of Ireland: a longitudinal investigation of trends over the period 2002-2014 using joinpoint analysis. BMC Health Serv Res 2018:18:474.

15 Lynch B, Fitzgerald AP, Corcoran P et al. Drivers of potentially avoidable emergency admissions in Ireland: an ecological analysis. BMJ Qual Saf 2019;28:438-48.

16 Thomas S, Foley C, Kane B et al. Variation in resource allocation in urgent and emergency care systems in Ireland. BMC Health Serv Res 2019;19:657

17 Foley C, Droog E, Boyce M, Healy O, Browne JP. Patient experience of different regional models of urgent and emergency care: A cross-sectional survey study. BMJ Open 2017;7:e013339.

18 Spurgeon PCM, Fulop N, Walters R et al. Evaluating models of service delivery: reconfiguration principles. London: National Institute for Health Research Service Delivery and Organisation programme, 2010.
19 Lynch K. INMO trolley watch analysis. Tableau, 2019 https://public.tableau.com/profile/kevin.lynch\#!/vizhome/ INMOTrolleyWatchAnalysis/INMOTrolleyWatch?publish=yes

20 Knowles E, Shephard N, Stone T, Mason SM, Nicholl J. The impact of closing emergency departments on mortality in emergencies: an observational study. Emerg Med J 2019;36:645-51.

21 Imison C, Sonola L. Insights from the clinical assurance of service reconfiguration in the NHS: the drivers of reconfiguration and the evidence that underpins it - a mixed-methods study. Health Serv Deliv Res 2015;3:9.

22 O'Cathain A, Knowles E, Maheswaran R et al. A system-wide approach to explaining variation in potentially avoidable emergency admissions: national ecological study. BMJ Qual Saf 2014;23:47-55.

23 Nally DM, Sørensen J, Valentelyte G et al. Volume and in-hospital mortality after emergency abdominal surgery: a national population-based study. BMJ Open 2019;9:11.

24 Cullen P. Better outcomes when emergency surgery performed by busier surgeons, study finds. The Irish Times 2019. www.irishtimes. $\mathrm{com} /$ news/health/better-outcomes-when-emergency-surgeryperformed-by-busier-surgeons-study-finds-1.4085907.

25 Parkhurst J. The politics of evidence: from evidence-based policy to the good governance of evidence. Routledge Studies in Governance and Public Policy. Oxford: Routledge, 2017.

Address for correspondence: Prof John Browne, School of Public Health, University College Cork, Western Gateway Building, Western Road, Cork, Ireland.

Email: j.browne@ucc.ie

\title{
Looking for jobs in medicine?
} Find your next hospital job

\author{
Find out more and subscribe to the \\ monthly Medicine Jobs bulletin at \\ www.rcplondon.ac.uk/jobsboard
}

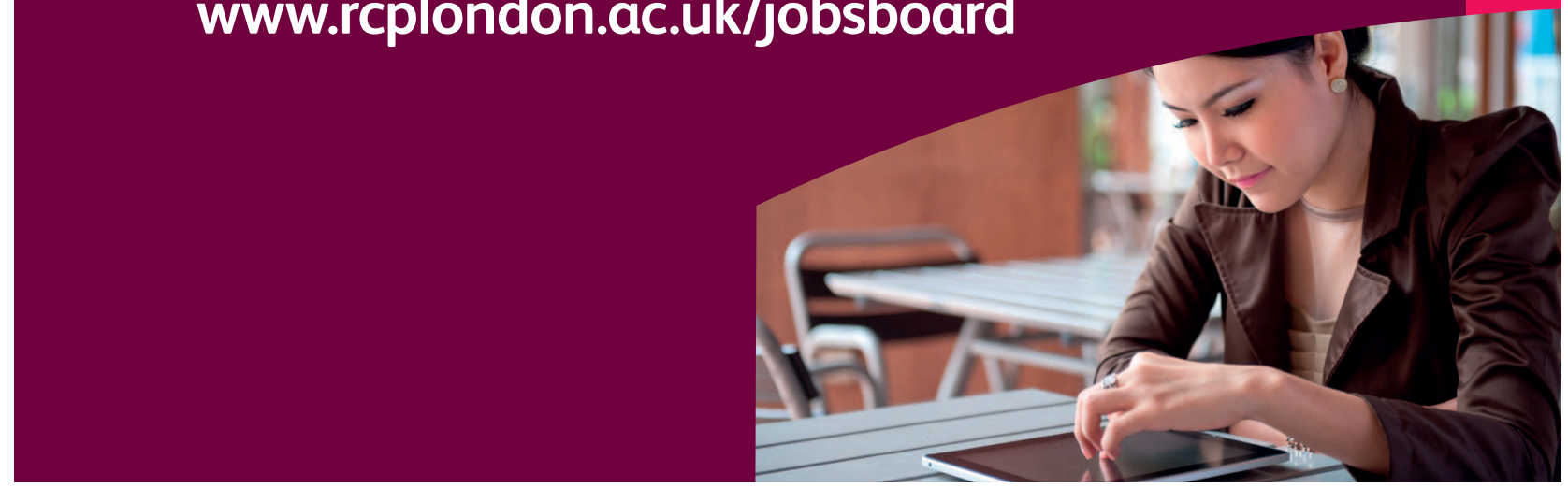

